\title{
Téoros
}

Revue de recherche en tourisme

\section{Transport aérien et marketing touristique}

\section{Michel G. Langlois}

Volume 6, numéro 1, mars 1987

Tourisme et transports

URI : https://id.erudit.org/iderudit/1080519ar

DOI : https://doi.org/10.7202/1080519ar

Aller au sommaire du numéro

Éditeur(s)

Université du Québec à Montréal

ISSN

0712-8657 (imprimé)

1923-2705 (numérique)

Découvrir la revue

Citer cet article

Langlois, M. G. (1987). Transport aérien et marketing touristique. Téoros, 6(1), 8-10. https://doi.org/10.7202/1080519ar d'utilisation que vous pouvez consulter en ligne.

https://apropos.erudit.org/fr/usagers/politique-dutilisation/ 


\title{
Transport aérien et marketing touristique
}

\author{
par Michel G. Langlois*
}

\section{Le transport aérien et le marketing du produit touristique}

Traditionnellement, le transport aérien est intimement lié au produit touristique situé au-delà d'un rayon d'action de 500 kilomètres du lieu de résidence du voyageur. Bien que le transport aérien soit une composante du produit touristique global, il demeure prédominant quant à l'importance qu'il occupe en terme de coûts. Que l'on parle de tourisme régional, national ou international, la variable transport aérien, qu'elle intervienne en rôle passif ou actif, aura un impact majeur sur le produit final. On ne peut imaginer un voyage outre-mer sans avion ou un voyage transcontinental sans qu'un transporteur aérien ne soit impliqué d'une façon ou d'une autre. Comment cette variable peut-elle être influencée? Quelles sont ses dépendances? Comment le gestionnaire en tourisme doit-il en tenir compte dans sa planification? Le présent article se veut une interrogation sur la corrélation entre le transport aérien et le produit touristique ainsi qu'une analyse de l'évaluation du transport aérien et de son impact réel sur le marketing du tourisme.

\section{L'évolution du transport aérien}

Ce n'est qu'au début des années ' 60 que le transport aérien dépassa le nombre de passagers maritimes. Une première qui allait tracer la route à un décloisonnement très important des cultures et à une évolution rapide du produit touristique. L'arrivée des avions réactés en 1957-58 avait déjà bousculé la productivité du transport aérien en doublant pratiquement la vitesse de croisière ainsi que la capacité des appareils. Désormais le transport aérien allait devenir non plus un produit de luxe, mais un produit accessible. Ce fut le début d'une explosion importante de sociétés aériennes nationales. On se souvient des grands réseaux des Pan Am, TWA et, plus près de nous, d'Air Canada, qui volaient à l'époque vers Moscou, Vienne, Prague, Bruxelles, etc. Le prix du pétrole représentait alors moins de $10 \%$ des dépenses. Dorénavant, il n'était plus nécessaire d'être

\footnotetext{
"Spécialiste du marketing dans lindustrie du transport
} aérien, docteur és sciences de la gestion.

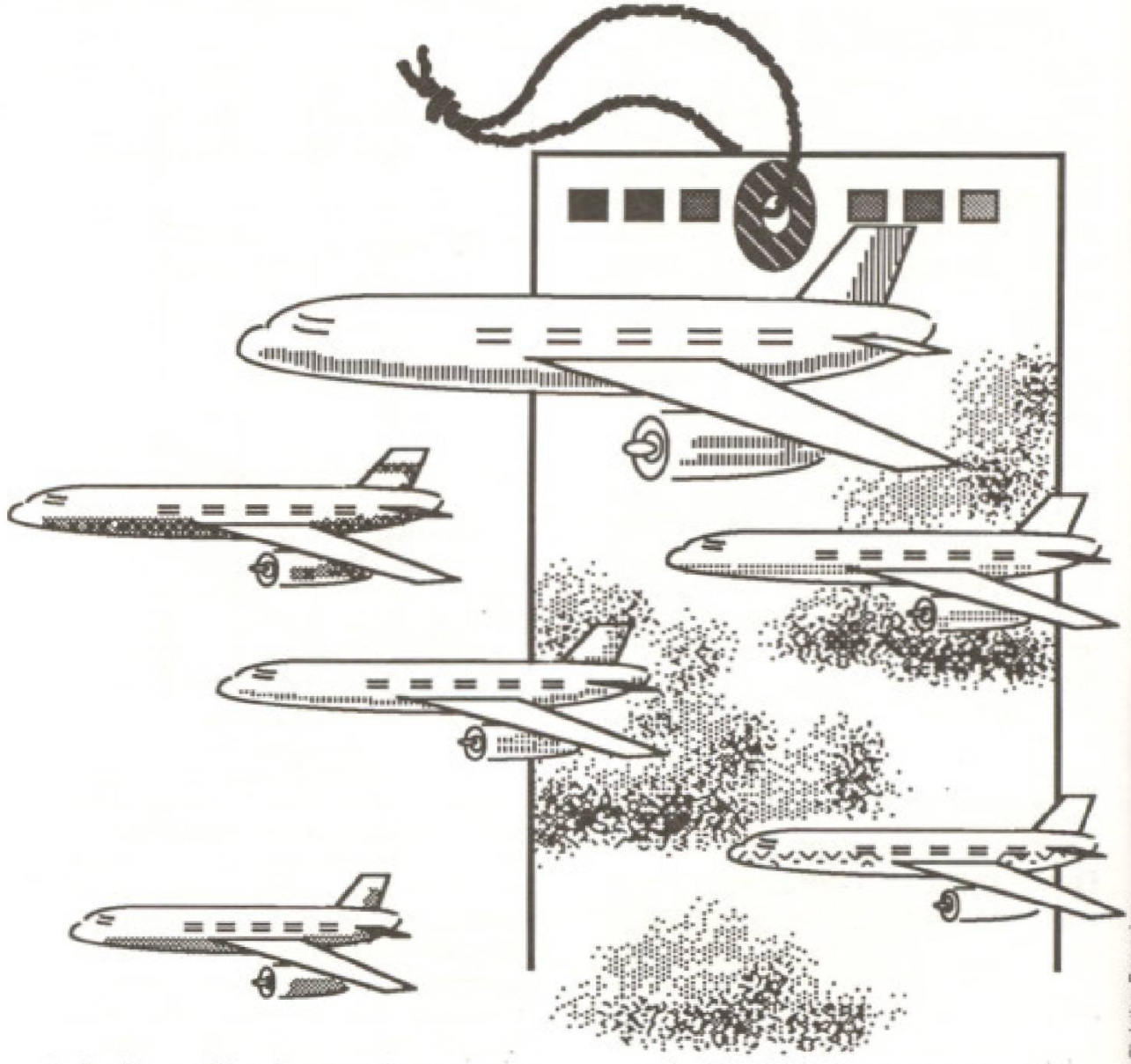

infirmière célibataire sans lunettes pour occuper un poste d'agent de bord. C'était la démocratisation du transport aérien. Une industrie où plus de $95 \%$ du trafic était régi par une réglementation de transporteurs réguliers, membres de l'LATA (International Air Transport Association). Le grand club fraternel où I'on établissait les normes de tarification et de règlementation. Le bouc émissaire que tous les transporteurs vênéraient afin d'éviter l'effondrement des tarifs. Deux transporteurs par route, un pour chaque pays. Une conjoncture de rêve: coûts peu élevés, marché en croissance, concurrence réduite et amicale, une rentabilité assurée avec des taux de remplissage dans les $50 \%$.

Ce contexte favorisa le développement de grandes structures bureaucratiques de relais technologiques où l'expertise technique était importée des trois grands manufacturiers Boeing, Douglas et Lockheed et diffusée en masse.

L'entrepreneurship et le leadership de ces manufacturiers sur l'industrie de l'époque les poussèrent à développer des modèles augmentant encore une fois la productivité. On se souviendra qu'entre 1965 et 1970 sont apparus les B-747, des DC-19 et des L-1011. Leur arrivée venait de donner un peu d'oxygène aux transporteurs dits réguliers dont le processus de bureaucratisation avait largement alourdi la structure de dépenses en venant réduire le coût par siège vendu, conservant ainsi en grande partie le leadership de la profitabilité chez le manufacturier. 
Puis soudain, la crise. Le grand effondrement. L'escalade du prix du pétrole fait remonter les coûts de carburant jusqu'à près de $30 \%$ des dépenses, projetant le seuil de rentabilité à des niveaux très élevés.

Les annees "70 furent pour les transporteurs aériens dits réguliers un cauchemar qui coutta la vie a certains, un peu plus tard.

Mais voilà que le leadership se déplaça du manufacturier d'avions à une nouvelle race d'entrepreneurs, les "Tour Operators" ou les voyagistes.

Comment aller chercher une clientèle de masse avec des prix et des coutts grandissants dans le transport et l'hôtellerie. La solution, des taux de remplissage très éleves 95 a $100 \%$. Comment y arriver? En créant un produit touristique global incluant l'hôtel et l'avion. Théoriquement ces taux de remplissage permettent une diminution des prix de 40 a $50 \%$ compte tenu des mêmes coûts. On assista donc à la naissance d'un nouveau système parallèle, les I.T.C. (Inclusive Tour Charters). Une révolution du produit touristique qui allait ouvrir la porte à une ère nouvelle dans l'industrie des voyages. En peu d'années, les transporteurs aériens dits réguliers virent leur part de marché passer de $100 \%$ à moins de $35 \%$. Tout d'abord, sur l'axe nord-sud, et plus tard sur l'Atlantique nord où ils réussissent à conserver une part plus importante du marché qui est composé en grande partie de voyageurs d'affaires. Vers les années 75-76, les transporteurs ditss "non réguliers" "étaient devenus plutôt " "réguliers" et la norme risquait de se déplacer brusquement n'eut été des actions importantes et drastiques que certains transporteurs tels Air Canada, mirent en place avec la création d'un nouveau concept d'offre sur les vols réguliers, soit les CBIT (Charter Bulk Inclusive Tours) qui ćtaient, à toute fin pratique, la vente de blocs de sieges au niveau tarifaire des charters assurant ainsi la viabilité des vols réguliers et des vols rentables.

Mais, il était quand même tard et les transporteurs dits régionaux de l'époque s'étaient taillés une bonne place sur les marchés internationaux. C'était la belle époque des PWA et Nordair et Quebecair qui sillonnaient les fins de semaines les cieux des Antilles, du Mexique et de l'Europe, en complément de leurs activités régionales et nordiques surtout concentrées durant la semaine. La base de marché, elle, s'élargissait et dorénavant le produit touristique long courrier était devenu accessible à tous.

Wardair, né de la grande révolution des "non réguliers" ", prit le leadership du produit touristique en prenant ses distances des voyagistes et en créant sa propre société de voyages. On assista alors à la troisième phase de leadership, soit celle du transporteur avec vacances par Wardair. Ce leadership s'étendit aux deux transporteurs

TABLEAU 1

Exemples d'origine d'entrepreneurship du produit touristique selon la forme

\begin{tabular}{|l|l|l|}
\hline \multicolumn{1}{|c|}{ FORME } & $\begin{array}{l}\text { TRANSPORT } \\
\text { AERIEN }\end{array}$ & $\begin{array}{l}\text { VOYAGISTE } \\
\text { (Tour operateur) }\end{array}$ \\
\hline $\begin{array}{l}\text { L'eniGiNepreneur a } \\
\text { capitaux prives }\end{array}$ & $\begin{array}{l}\text { Worldways } \\
\text { Nationair }\end{array}$ & $\begin{array}{l}\text { Tours Mont Royal } \\
\text { Jet Vacances } \\
\text { Trallic } \\
\text { Solvac }\end{array}$ \\
\hline $\begin{array}{l}\text { L'entrepreneur a } \\
\text { capitaux publics }\end{array}$ & $\begin{array}{l}\text { TWA-CP Air } \\
\text { Wardair } \\
\text { Eastern }\end{array}$ & CP Holidays \\
\hline L"Etat entrepreneur & $\begin{array}{l}\text { Air Canada } \\
\text { Air France } \\
\text { Aero Mexico } \\
\text { Dominicana }\end{array}$ & $\begin{array}{l}\text { CIT - Touram } \\
\text { Jet Tour - Intourist }\end{array}$ \\
\hline $\begin{array}{l}\text { Le consortium } \\
\text { d"États }\end{array}$ & $\begin{array}{l}\text { Liat } \\
\text { Air Afrique }\end{array}$ & \\
\hline
\end{tabular}

dits "réguliers" qui, à l'instar des "non réguliers"' finalement, emboitèrent le pas avec la création de Touram chez Air Canada et CP Holidays chez CP Air. Ces derniers d'ailleurs copiant gentiment les actions stratégiques déjà entreprises par feu Nordair avec Treasure Tours et PWA Holidays qui affirmait un leadership qui ne fit que s'acentuer par la prise de contrôle récente des réseaux CP Air, EPA, Nordair, Quebecair et Nordair Metro.

Une longue route pour finalement donner aux transporteurs á́riens dits "réguliers" un leadership qui aurait dû se manifester il y a bien longtemps. Il est bien de rendre à César ce qui appartient à César, à condition que Cesar ne s'endorme plus au combat et mette fin à la myopie commerciale.

\section{Les variables influençant le transport aérien et le marketing du tourisme}

\section{a) L'origine et la forme}

d'entrepreneurship

Si l'avancement de l'industrie du transport aérien est un balancier entre le manufacturier d'avions, les transporteurs et les tours operateurs, l'entrepreneurship du produit touristique lui, sera caractérisé par sa forme d'une part et son origine d'autre part. Voir tableau I. C'est ainsi que cet entrepreneurship pourra être dirigé par le transporteur, qu'il soit horaire fixe ou nolise, desirant augmenter son achalandage ou par des voyagistes ou tours opérateurs, qui prendront l'initiative de fabriquer un produit a l'aide d'un transporteur à horaire ou nolises. Cet entrepreneurship pourra être à capitaux privés ou publics; il est cependant assez fréquent dans cette industrie de voir l'État ou un consortium d'États se substituer à I'entrepreneur privé afin d'équilibrer sa balance touristique ou carrément sa balance commerciale, ou pour assurer un développement économique de sa région ou son pays. A priori, aucune formule semble démontrer une plus grande efficacité que l'autre. Une étude empirique, structurée et approfondie pourrait, par contre, infirmer ou confirmer cette observation. b) Les imperatifs saisonniers et climatiques

Ces deux variables souvent interteliees ont un impact sếrieux quant au choix des touristes. L'être humain recherche naturellement une température et un climat plus cléments. C'est ainsi que les habitants de l'hémisphère nord vont plutốt voyager sur l'axe nord-sud durant l'hiver et l'axe estouest durant la saison d'été avec une plus grande concentration durant les quatre mois où l'ecart climatique est le plus accentué. Deux grandes stratćgies sont utilisées par les transporteurs et les voyagistes pour maintenir un minimum d'activités durant les mois creux. La première est la baisse tarifaire et la deuxième le developpement de groupes d'intérêts particuliers tels les congres et les voyages de motivation. Phénombne assez cocasse sur l'Atlantique nord, la pratique de tarifs réduits pour deplacer la pointe des mois de juillet et aoult à trop bien réussi, faisant des mois de mai et juin des héros au détriment du mois d'aoùt et provoquant surtout une baisse tarifaire générale entraînant des recettes globales plutôt anémiques pour les transporteurs. D'autre part, la baisse de tarifs d'êté vers la Floride genère une clientèle familiale qui ne pourrait en haute saison profiter de cette destination.

\section{c) Les affinités culturelles}

Les affinités culturelles jouent un rôle déterminant dans le choix des destinations touristiques. Pourquoi les résidents du Québec préféreront davantage l'entrée en Europe par la France plutôt que l'Angleterre alors que les résidents de l'Ontario préféreront Londres en Angleterre. Pourquoi le Mexique est-il tres populaire au Québec et la Barbade ou la Jamaïque en Ontario? Faut-il forcer des destinations aux marchés ou identifier les produits répondant davantage au marche? Pourtant, les Allemands aiment bien l'Espagne et les Français la Grèce. En quoi le Canada ou l'Amérique du Nord different-ils? Seraitce lié a la quantité de choix disponibles aux Nord-Américains par rapport aux Européens, particulièrement en ce qui concerne les destinations soleil à prix abordables? 
d) Concurrence globale internationale Dans le domaine touristique, chaque pays a tendance à avoir une orientation produit et croire en l'unicité et la supériorité de sa destination. Cependant, chaque destination et chaque pays sont observés par le marché international â l'intéricur d'un système complexe de possibilites.

Comme tout produit commercialise, il est important de toujours conserver son avantage différentiel d'une part, et de s'assurer que la destination demeure concurrentielle. Les Antilles françaises ont eu un problème majeur de pricing en persistant à offrir des forfaits incluant les repas, augmentant ainsi leur prix d'appel et en les plaçant hors marché quant aux prix. Le raisonnement des hôteliers souhaitant conserver les clients chez eux pour tous les repas les a amenés à perdre des clients pour d'autres destinations. Ils avaient oubliê que leur concurrent n"était pas nécessairement l'hôtelier voisin mais peut-être l'ile voisine.

\section{e) Climat politique}

La sécurité joue un rôle prépondérant dans le déplacement des touristes qui sont au départ suffisamment insécures en quittant leurs pays ou leur ville pour une autre destination. Le climat politique est souvent une variable imprévisible qui peut affecter une destination et ses fournisseurs de services touristiques d'une façon majeure et en laissant souvent des traces permanentes. Comment une destination peut-elle neutraliser un climat politique momentanément troublê? Comment l'Europe et particulièrement la France ont-ils reconquis leur clientèle insécurisée par le terrorisme? Comment Haïti se remet-elle du départ de JeanClaude Duvalier? Pourquoi les gens ont recommencế à aller en Jamaïque? Le succès d'un retour sur le marché semble de prime abord lié à l'habileté à garantir la sécurité et l'encadrement du touriste. La promotion des clubs tous services compris a permis à la Jamaïque de se redonner une crédibilité après plusieurs années de difficultés touristiques. Il y aurait peut-être là des éléments de neutralisation des obstacles liés à un climat politique temporairement affecté.

\section{f) Disponibilité d'avion}

Le parc aérien d'un pays est limité aux capacités des transporteurs de ce pays ainsi qu'à celles des pays des destinations ayant les droits d'atterrissage. Or, il est intéressant de constater que l'ajout d'une capacité additionnelle a deux impacts majeurs sur la performance du produit touristique. La première est liêe au jeu de l'offre et de la demande et c'est ainsi que l'ajout d'une capacité massive entraine une réduction des prix aux voyagistes et éventuellement aux consommateurs puisque le taux de remplissage semble au depart moins garanti. Le voyagiste ou le transporteur ayant les nerfs les plus solides tiendra son prix alors que l'autre risquera le tout pour le tout et pren- dra l'initiative de baisser les prix en souhaitant gagner le maximum de passagers avant que ses compétiteurs le suivent dans la spirale descendante des tarifs. Nous avons assisté à ce phénomène sur l'Atlantique nord avec l'augmentation de la capacité sur la route entre Montréal et Paris par Worldways, Nordair, Nationair, Quebecair et Minerve en plus d'Air Canada et d'Air France.

Un deuxième phénomèné intéressant avec l'augmentation massive de la capacité est la naissance de nouvelles destinations plus attrayantes et plus originales afin de s'attirer davantage de passagers que leurs compétiteurs. C'est ainsi que sont apparues sur le marché québécois avec Nationair des destinations telles que Rio ou Recif au Brésil et avec Quebecair Margarita et Barcelona au Venezuela.

\section{g) Les infrastructures aéroportuaires}

Les limites aéroportuaires d'une destination peuvent empêcher le développement touristique, soit en n'ayant pas la bonne longueur de piste. C'est le cas des Iles de la Madeleine qui représentent une destination touristique intéressante mais où les réactés ne peuvent atterrir à cause d'une piste limitée. Les heures d'ouverture de l'aéroport peuvent empêcher d'effectuer des vols de nuit vers une destination donnée avec un couvre-feu, soit sécuritaire pour prévenir des attaques insolites (Haiti) ou pour le confort des residents dans l'entourage de l'aéroport. Un dernier élément est liế à la classification de l'aéroport au rang de domestique, c'est-à-dire sans facilites de douanes. C'est ainsi que des vols entre le Saguenay et la Floride furent impossibles bien qu'un marché était présent.

\section{h) Les infrastructures d'accueil}

En plus d'un site exceptionnel et d'un aéroport d'entrée, les destinations touristiques doivent être en mesure d'offrir la capacité d'hébergement et l'activité nécessaire à maintenir un niveau d'interrêt constant pour la clientèle. C'est ainsi que les Iles Turks et Caicos furent difficilement commercialisables avant l'ouverture d'un club Méditerranéen puisque malgré son site et ses plages exceptionnelles, aucun attrait hôtelier et aucune activité ne faisaient ressortir cette destination des autres. Une destination telle que la Floride offre avec son infrastructure d'accueil et sa capacité d'encadrement un exemple parfait de l'impact de l'infrastructure d'accueil.

\section{i) La réglementation}

Traditionnellement, le transport aérien était une industrie réglementée. L'obtention de routes était difficile et les droits acquis glorieux. La création des règlements de nolisement faciles de mise en application a levé une barrière presqu'hermétique sur une industrie plus ou moins amorphe. A toute fin pratique, il n'existe plus de contraintes réelles sur les marchés nationaux et inter- nationaux permettant ainsi à tout entrepreneur de risquer l'aventure comme voyagiste ou comme transporteur. Cette variable n'a plus le poids qu'elle possédait il y a quelques années et est en réalité nulle comme influence sur la décision de mise en place d'un produit touristique.

\section{j) Tarification et coûts}

Le prix de vente aux consommateurs a joué un rôle primordial dans l'explosion de ce trafic. La mise en place des programmes de vols nolisés a été une révolution fondamentale de l'industrie, la surcapacité a contribué à orienter ce phénomène. La rationalisation graduelle de l'industrie, avec la disparition de certains transporteurs et les grandes difficultés des autres, laisse présager que la chute des prix ou leur maintien à un niveau bas ne sera pas éternelle et, à moins d'une révolution technique réduisant le coût par siège/mille aérien ou une baisse drastique du pétrole, des hausses de prix sont à l'horizon pour les touristes. Quel sera alors l'impact sur le volume total? Quelle est 1'élasticité de ce marché qui est maintenant habitué à voyager? Quels produits seront alors substitués pour permettre de maintenir ces voyages? Oủ se situent les voyages dans la priorité des gens?

\section{k) La dépendance des destinations} Bien que plusieurs variables influencent le produit touristique et une de ses composantes majeures, le transport aérien, certaines destinations n'ont pas de choix et doivent s'accommoder de toutes les turbulences causées par ces variables. La raison: leur isolement. C'est le cas de toutes les destinations insulaires au départ, mais en réalité, toute région désirant s"attirer des visiteurs devient dépendante de ces variables et il nous apparaitt important d'en comprendre la portée afin de réduire cette dépendance et maintenir un niveau d'activité ́cconomique acceptable.

\section{Conclusions et perspectives de recherche}

Les informations énoncées plus haut sont le résultat d'observations pratiques et deviennent des bases interessantes pour des recherches plus approfondies afin d'aider les intervenants du milieu touristique à sajsir les données ayant les plus grandes portées d'action. Assisterons-nous à un nouveau leadership ou verra-t-on le balancier de la productivité et de la performance de retour dans la cour des manufacturiers d'avions? Peut-on encore rationaliser le transport aérien avec la technologie actuelle? $\mathrm{Y}$ a-r-il une place pour le privé dans le transport aérien ou devrait-on laisser l'État agir comme entrepreneur en assumant les pertes que le privé ne peut assumer? Voilà un dilemme auquel les faillites ou difficultes financières de la plupart des transporteurs américains, incluant les plus solides et les plus innovateurs tels People Express, nous invitent à réfléchir. $f$ 